Highly Energetic Physical Processes and

Mechanisms for Emission from Astrophysical Plasmas

IAU Symposium, Vol. 195, 2000

P. C. H. Martens, S. Tsuruta, and M. A. Weber, eds.

\title{
General-Relativistic MHD Simulation of Jets from a Geometrically Thin Accretion Disk Around a Schwarzschild Black Hole
}

\author{
S. I. Aoki \\ Department of Astronomy, University of Tokyo, Bunkyo-ku, Tokyo \\ 113-0033, Japan
}

S. Koide

Faculty of Engineering, Toyama University, Gofuku, Toyama 930, Japan

K. Shibata

Kwasan and Hida Observatories, Kyoto University, Yamashina, Kyoto 607-8471, Japan

T. Kudoh

Solar Physics Division, National Astronomical Observatory, Mitaka, Tokyo 181-8588, Japan

\begin{abstract}
We have performed a 2.5D, nonsteady, general-relativistic MHD simulation. Initially, we assumed a uniform magnetic field, a geometrically thin accretion disk rotating at Keplerian velocity, and a hydrostatic corona around a Schwarzschild black hole. We have investigated the formation mechanism of gas-pressure driven jets expected by Koide et al. and found the strong dependence of jet velocities $\left(\gamma_{j}=1 / \sqrt{1-V_{j}^{2}}\right.$; Lorentz factor of jets) on the ratio of the density of the accretion disk to that of the corona $\left(\rho_{d} / \rho_{c}\right)$, where $\gamma_{j}^{2}-\gamma_{j} \propto\left(\rho_{d} / \rho_{c}\right)^{0.75}$.
\end{abstract}

\section{Introduction}

Many numerical MHD simulations have been performed to explain astronomical jets (e.g., Uchida \& Shibata 1985; Shibata \& Uchida 1986). Among these jets, AGN jets have a very high velocity (nearly the speed of light), and there is evidence of the existence of supermassive black holes $\left(\sim 10^{8} M_{\odot}\right)$ in the central regions of AGNs. Hence, general-relativistic effects are important in the formation of AGN jets, and a general-relativistic MHD (GRMHD) simulation must be carried out to model AGN jets. Koide, Shibata, \& Kudoh (1998) have succeeded in performing a nonsteady, GRMHD simulation of jets for the first time. In their results, jets have 2-layered structures which consist of magnetically driven jets and gas-pressure driven jets. The latter jets are characteristic features in their GRMHD simulation. Their velocities reach about $90 \%$ of the speed of light. In this paper, we have investigated the properties of gas-pressure driven jets 
(a)

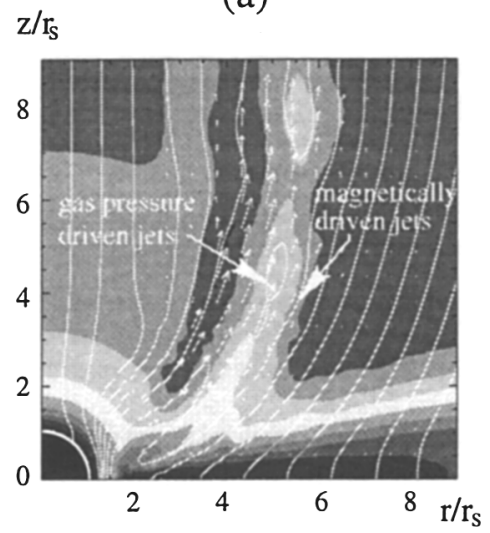

(b)

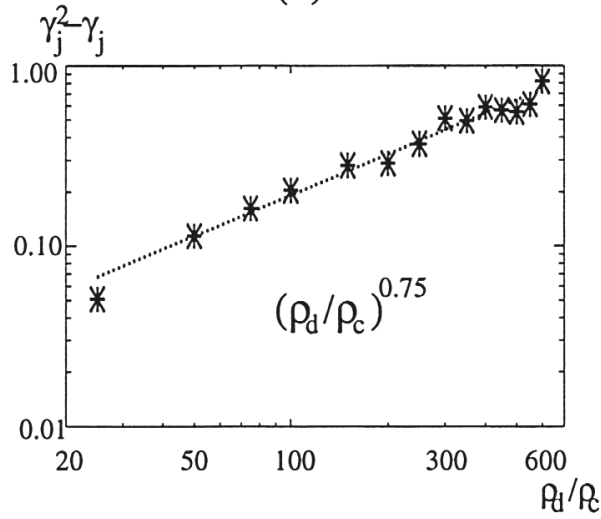

Figure 1. (a) Density (grey scale), magnetic field lines (white lines), and poloidal velocity vectors (arrows) are shown. There are a black hole (black quarter circle at lower-left corner) and an accretion disk (the high-density region at the equatorial plane). The unit of length is $r_{s}$, the Schwarzschild radius. (b) The velocity (Lorentz factor; $\gamma_{j}$ ) dependence on the ratio of the density of the accretion disk $\left(\rho_{d}\right)$ to that of the corona $\left(\rho_{c}\right)$. The abscissa is $\rho_{d} / \rho_{c}$, and the ordinate is $\gamma_{j}^{2}-\gamma_{j}$.

in detail by performing a GRMHD simulation for the case of a Schwarzschild (nonrotating) black hole.

\section{Results}

Figure 1a shows the density contour plot after about one rotation of the Keplerian disk at $(r, z)=(3,0)$. Magnetically driven jets are accelerated by the magnetic force. On the other hand, gas-pressure driven jets are accelerated by the gas-pressure force enhanced by the shocks (at about $(r, z)=(2,0)$ ) formed in the accretion flow from an accretion disk to a black hole. The velocity of the latter jets are much higher than those of the former jets, in our results. To explain the formation mechanism of AGN jets, we have investigated the properties of gas-pressure driven jets. Figure $1 \mathrm{~b}$ shows the dependence of the velocity of gas-pressure driven jets $\left(\gamma_{j}\right)$ on $\left(\rho_{d} / \rho_{c}\right)$. The dotted line is obtained by leastsquare fitting of simulation results (star symbols). In this figure, $\gamma_{j}^{2}-\gamma_{j}$ means specific kinetic energy in the Newtonian case $\left(\frac{1}{2} V_{j}^{2}\right)$, and it shows the dependence of $\gamma_{j}^{2}-\gamma_{j} \propto\left(\rho_{d} / \rho_{c}\right)^{0.75}$. We can explain this dependence by considering the formation mechanism of gas-pressure driven jets.

\section{References}

Koide, S., Shibata, K., \& Kudoh, T. 1998, ApJ, 495, L63

Shibata, K., \& Uchida, Y. 1986, PASJ, 38, 631

Uchida, Y., \& Shibata, K. 1985, PASJ, 37, 515 